\title{
Ethnic hair: Thermoanalytical and spectroscopic differences
}

\author{
Marc A. Oliver (D) | Luisa Coderch | Victor Carrer | Clara Barba (i) | Meritxell Marti
}

Institute of Advanced Chemistry of Catalonia, IQAC-CSIC, Barcelona, Spain

Correspondence

Clara Barba, Institute of Advanced Chemistry of Catalonia, IQAC-CSIC, Barcelona, Spain.

Email: cbaesl@iqac.csic.es

\begin{abstract}
Background: The aim of this study is to characterize and detect the possible differences among the hair of three different ethnicities: African, Asiatic and Caucasian.

Materials and Methods: The differences in water adsorption/desorption behaviour of hairs were studied using a thermogravimetric balance and compared with the analysis of the lipid distribution and order using synchrotron-based Fourier transform infrared microspectroscopy. Besides, the thermal thermogravimetry (TG) and differential scanning calorimetry (DSC) analyses on human hair were executed.

Results: Differences in the diffusion coefficients were evidenced. African hair exhibited increased permeability. Caucasian hair displayed a higher water absorption capability with increasing humidity but with a slow diffusion rate. The Asian fibre appeared to be more resistant to hydration changes. The spectroscopic analysis showed notable differences in the cuticle lipids. The African cuticle exhibited more lipids with a lower order bilayer. The outmost layer of Caucasian fibres contained more ordered lipids, and the Asian fibres show a very low level of lipids on the cuticle region. The DSC results indicate no difference in the thermal stability and TG showed higher water content in the Caucasian fibre and a possible lower cysteine disulphide bond content in the African hair matrix.

Conclusion: The triple approach demonstrated the permeability differences among the ethnic fibres and their correlation with the properties of their cuticle lipids. These differences could have particular relevance to the hair care cosmetic market.
\end{abstract}

\section{KEYWORDS}

chemical analysis, hair growth/hair biology, lipid, moisture content, spectroscopy

\section{1 | INTRODUCTION}

Hair is a protein fibre (85\%) and contains low levels of lipids (1\%-5\%) and water (10\%). Hair consists of a follicle and a shaft. ${ }^{1}$ The shaft, the part of the hair that is visible above the skin, has a width of 50-100 $\mu \mathrm{m}$ and is composed of three main parts: the medulla, the cortex and the cuticle. The medulla, which possesses a diameter of 5-10 $\mu \mathrm{m}$, is composed of loosely packed cells and is not always presented in the hair. The cortex, which is $45-90 \mu \mathrm{m}$ thick, accounts for $90 \%$ of the total hair mass. Seven to ten tetramer spiral units, known as intermediate filaments, compose the microfibrils of the cortex. The intermediate filaments are surrounded by a matrix of high-glycine/tyrosine proteins. The proteins interact with the intermediate filaments through intermolecular disulphide bonds that provide inertness, rigidity and high mechanical strength to the hair fibres. ${ }^{1,2}$ The cuticle, at the surface of the hair, has a 5 - $\mu \mathrm{m}$-thick protective covering consisting of superimposed layers of flat overlapping structures. ${ }^{3,4}$ Low levels of lipids form part of the intercellular membrane complex, and these lipids are presented in the cortex and in the cuticle. In addition, the hair medulla also contains a fraction of rich lipids. ${ }^{5,6}$ The presence of cuticles is fundamental to maintain the internal water content of the 
hair. Therefore, internal hair lipids can amplify the hair's barrier function to prevent external substances from going into the hair fibres. ${ }^{3}$

Virgin human hair accounts for an important portion of the phenotypic variation among the different human races. Typically, three different types of ethnic hair can be categorized: African, Caucasian and Asian. There are numerous studies analysing some of characteristics related to the human skin but very few on the hair morphology across populations. These studies have focused primarily on hair colour, diameter and section, the shape of the fibre and its responses to external stimuli, which have been used to determine certain mechanical properties. ${ }^{7}$ The proteins and amino acids that constitute keratin are similar in the three ethnic groups. ${ }^{6,8}$ Lipid composition was previously studied, and lipid extraction revealed increased levels of total lipids in African hair compared with Asian and Caucasian hair. These lipids could be derived from an external sebaceous origin, which are presented at relatively high levels in African hair fibres. Asian and Caucasian hair fibres present similar fractions of external lipids with higher extractions of internal lipids from the hair matrix cell. ${ }^{9}$

Water alters the properties of human keratin fibres and, therefore, plays an important role in its cosmetic performance. ${ }^{10}$ Dynamic vapour sorption (DVS) analysis can characterize the response of hair to humidity changes. The relationship between the equilibrium moisture content and relative humidity $(\mathrm{RH})$ at a constant temperature is known as the sorption isotherm. Surface absorption/desorption and dehydration/hydration are the typical responses. In surface adsorption, water can be slightly linked to the surface by van der Waals forces via physisorption, or more strongly linked via chemisorption. While chemisorption is generally considered irreversible, physisorption is reversible via humidity reduction or increasing temperature. ${ }^{11}$

Altering the water sorption isotherm by isothermally applying small changes in the cumulative humidity allows for static and dynamic analysis, from which the equilibrium and diffusion coefficients of the water content are deduced. ${ }^{12}$ Keratin's moisture sorption isotherms have been the subject of several works, and models have been specially developed to describe the shape of the moisture adsorption and desorption isotherms. The role of the glass transition in generating the sigmoidal shape of the adsorption isotherm was fully studied in the Vrentas and Vrentas model. ${ }^{13}$ The uptake of water by polar polymers was also described using the Flory-Huggins equation. ${ }^{14}$ The Guggenheim, Andersen and de Boer (GAB) sorption equation also provides monolayer sorption values that present a much wider range of relative vapour pressure intervals than the BET equation. ${ }^{15}$ The GAB and BET isotherms are closely related as they are based on the same statistical model. The GAB model is an improvement of the BET model and shares two of the original BET constants: (a) $W_{m}$, the monolayer capacity and (b) $C_{g}$, the energy constant.

With the aim of elucidating the different hydric behaviours of the fibres, some studies have been performed to identify the role of lipids on the fibre properties. ${ }^{16,17}$ Studies show that lipids play an important role in the water permeability of hair fibres and changes in water permeability are related to the amount of lipids and the order in the cuticle region. Changes in the lipidic properties could modulate the water dynamics of the fibre. ${ }^{18}$ To this end, the cuticle lipid properties will be evaluated by synchrotron-based Fourier transform infrared microspectroscopy ( $\mu$-FTIR). The combination of a conventional infrared spectrometer with a synchrotron infrared source and microscope allows for a spatial determination of the chemical composition of the samples, and several studies have been performed using $\mu$-FTIR. ${ }^{19,20}$ Differences in the lipid order or amount will be studied by a combination of the microscopy transmittance FTIR with an ALBA synchrotron infrared light source. Previously, several studies have described the lipid properties of hair fibres using FTIR analysis. ${ }^{19,21-23}$ However, no study has described the quantities and order of cuticle lipids in African, Asian and Caucasian hairs, as well as their water dynamics properties.

Thermal analysis techniques have been widely used to characterize hair samples, and extensive studies have investigated the thermal behaviour, dehydration/hydration effects, melting behaviour of the $\alpha$-crystallites and the kinetic study of hair. ${ }^{24-28}$ According to Wendlandt, ${ }^{29}$ the most commonly thermoanalytical techniques used are differential scanning calorimetry (DSC), thermogravimetry (TG) and differential thermogravimetry (DGT). DSC is a thermal analysis technique in which energy differences are measured according to a substance and a thermally stable reference material as a function of temperature; thus, exothermic or endothermic events are detected. ${ }^{29}$ Differential scanning calorimetry (DSC) data have been used to study the thermal behaviour of hair ${ }^{24}$ and to detect whether cosmetic treatments could repair damaged hairs; for example, decreasing peak temperatures may indicate structural changes and chemical damage. ${ }^{30}$ TG provides information about the changes in the mass as a function of time and/or under certain temperature-atmosphere conditions. The curves obtained provide information on the composition and thermal stability of the sample, intermediate products and residues formed. ${ }^{29}$ Additional research on the thermal stability of hair has been DSC coupled with TGA. As the TG detects events associated with mass loss, the DSC indicates the thermal events related to mass variations. ${ }^{25}$ Several authors, who focused on the thermal analysis of human hair, executed TG and DSC analyses with the purpose of characterizing the keratin of human hair ${ }^{27,28}$ and verifying the effects of hair care products on the keratin structure. ${ }^{25,26}$ Lately, these techniques have been applied to differentiate the hair samples of different ethnicities ${ }^{27}$; however, slight variations were found. Further work must be done in this line of research supported by the techniques previously mentioned.

The aim of this study is to detect and characterize the possible differences among three different ethnicities hairs: African, Asiatic and Caucasian. In previous studies, the lipid differences between the ethnicities and the relevance of the cuticle lipids in the fibre properties has been reported. Consequently, the characteristics of the lipids in the outmost layer and their implications with the properties of the fibres were evaluated. Different techniques were used, including water sorption and desorption analysis and synchrotron-based $\mu-F T I R$, to elucidate the possible differences in the hair structure and permeability. Furthermore, TGA and DSC were applied to assess 
the thermal properties of the hair samples. The combination of the various techniques could be effective in characterizing the hair types among ethnicities.

\section{2 | MATERIALS AND METHODS}

\section{1 | Hairs}

The hair samples used in this study were Natural Virgin Brown Caucasian Hair and Natural Dark Brown Asiatic Hair without pretreatment and Natural Kinked Afro Hair with a thermal pretreatment. All hair samples were provided by the De Meo Brothers.

The hair samples were washed with $3 \%$ diluted Pantene Pro-V commercial shampoo (Procter \& Gamble) at a hair/surfactant 1/30 bath ratio followed by a thorough rinse with water and drying at ambient conditions.

\subsection{Determination of dynamic water vapour sorption}

The hair samples were cut into short pieces of approximately $3 \mathrm{~mm}$ and placed in a holder. A thermogravimetric balance Q5000SA Sorption Analyzer (TA Instruments) with a controlled humidity chamber was used to obtain the absorption and desorption values. The hair samples weighed between 9 and $12 \mathrm{mg}$. The experiments were conducted with a total gas flow of $200 \mathrm{~mL} / \mathrm{min}$, at $25^{\circ} \mathrm{C}$, following a measuring procedure ${ }^{17}$ : An initial drying at $60^{\circ} \mathrm{C}$ and $0 \%$ $\mathrm{RH}$ overnight, then, a pre-stabilization at $25^{\circ} \mathrm{C}, 0 \% \mathrm{RH}$, followed by initial absorption kinetics at $5 \% \mathrm{RH}$. In the Absorption curve, the sample was subjected to absorption tests that increased in steps of $10 \%-95 \%$. The sample remained in each step until its mass reached equilibrium. Equilibrium was arbitrarily defined as a change in mass of $<0.02 \%$ per minute for 10 minutes. In the Desorption curve, the sample previously stabilized after the absorption process at $95 \% \mathrm{RH}$, was subjected to desorption decreasing steps of $10 \%-5 \% \mathrm{RH}$. The sample was then stabilized at $5 \% \mathrm{RH}$. As in the absorption curve, the sample remained in each stage until its mass equilibrated. The degree of hysteresis for the hair samples was calculated by subtracting the mass at the desorption and absorption of the same humidity. Each hair sample was evaluated in triplicate.

The GAB mathematical model describes the sorption isotherms with a theoretical basis, and its parameters provide a more physical aspect to the sorption process compared to other empirical models. The monolayer moisture concept is the basis of the GAB model and provides a value for the monolayer moisture content of the material. ${ }^{31}$ In the present work, the sorption isotherm data were modelled following the GAB model, consistent with previous work. ${ }^{32}$ The GAB equation and the parameters used to fit the experimental absorption/desorption data are listed in Table 1. The coefficient of the determination $\left(R^{2}\right)$ was also evaluated to determine the strength of the model.
The minimum values of the GAB model derivative and the equilibrium moisture content in relation to $\mathrm{RH}$ correspond to the points where the multilayer sorption starts to dominate over the monolayer sorption, $a_{\mathrm{w}}$. The moisture content at this point is $W_{\mathrm{c}}$. With the GAB parameters, the number of secondary layers can be calculated using Equation $2 .^{33}$

When the moisture absorption/desorption tests are performed with hygroscopic samples, the equilibration takes a very long time, although the greatest interchange of water occurs during the initial steps. Therefore, to shorten the testing time, some conditions were fixed. Although equilibrium was not reached, the final regain $\left(R_{f}\right)$ in each step was used to calculate the apparent diffusion coefficients.

The method applied by Vickerstaff ${ }^{34}$ to study the diffusion of dyes within fibres was used to obtain the diffusion coefficient. The method is represented by an expression derived from the application of Fick's equation to moisture diffusion. Satisfactory results were obtained for the early stages of the moisture absorption with this expression, as in the case of the dye diffusion. The fraction of absorbed water plotted against the square root of the absorption time, should lie on a straight line in which the slope is the square root of the apparent diffusion coefficient, $D_{\mathrm{a}}$. The apparent diffusion coefficient is measured in $\min ^{-1}$ when it is measured over the sample's mass.

$$
R(t) / R_{\mathrm{f}}=\sqrt{D_{\mathrm{A}}} \sqrt{t}
$$

\section{3 | Thermogravimetric analysis}

Thermogravimetric analysis was performed with a TGA instrument (Model TGA/SDTA $851^{\mathrm{e}}$, Mettler Toledo). Approximately $5 \mathrm{mg}$ of cut hairs was packed into an aluminium pierced pan $(100 \mu \mathrm{L})$ and heated under a nitrogen stream from 25 to $900^{\circ} \mathrm{C}$ at a heating rate of $10^{\circ} \mathrm{C} /$ $\min$.

\section{4 | Differential scanning calorimetry measurement}

Differential scanning calorimetry was performed using a Mettler Toledo DSC Model DSC-821 equipped with a Dewar containing liquid nitrogen. The sample $(6-7 \mathrm{mg}$ ) of snipped hair was placed in a sealed microperforated aluminium pan $(50 \mu \mathrm{L})$ and heated under a nitrogen stream $(50 \mathrm{~mL} / \mathrm{min})$ from $20-25$ to $275^{\circ} \mathrm{C}$ at a heating rate of $10^{\circ} \mathrm{C} / \mathrm{min}$.

\section{5 | Synchrotron-based Fourier transform infrared microspectroscopy ( $\mu$-FTIR) and data acquisition}

Hair tufts of $1 \mathrm{~cm}$ were embedded into an optimal cutting temperature (OCT) compound (Bright Instruments) and immediately frozen using liquid N2. Sample blocks were cut in $5 \mu \mathrm{m}$ cross sections using a Cryostat CM3050 S (Leica Biosystems Nussloch). Sections were placed on CaF2 circular windows, with a $1 \mathrm{~mm}$ thickness and $13 \mathrm{~mm}$ diameter (Crystran). 
TABLE 1 Parameters used in the GAB model to fit the experimental sorption data

\begin{tabular}{|c|c|c|}
\hline \multicolumn{3}{|c|}{ Mathematical equations } \\
\hline $\mathrm{GAB}^{33}$ & $W=W_{\mathrm{m}} \cdot C_{\mathrm{g}} \cdot K \cdot a_{\mathrm{w}} /\left[\left(1-K \cdot a_{\mathrm{w}}\right) \cdot\left(1-K \cdot a_{\mathrm{w}}+C_{\mathrm{g}} \cdot K \cdot a_{\mathrm{w}}\right)\right]$ & (1) \\
\hline Layers $\mathrm{n}^{\circ}$ & $W_{1}=\left(W_{m} \cdot C_{g} \cdot K\right) /(1-K) /\left(1-K+C_{g}\right) \rightarrow$ layers $n^{\circ}=W_{1} / W_{m}$ & (2) \\
\hline \multicolumn{3}{|c|}{ Parameters } \\
\hline$a_{\mathrm{w}}$ & \multicolumn{2}{|l|}{ Water activity, as vapour relative pressure $p / p_{0}$, in which $p_{0}$ is the saturated vapour } \\
\hline$W_{c}$ & \multicolumn{2}{|c|}{ Water moisture at critical point, where secondary layers predominate over monolayer ( $\mathrm{g}$ sorbed/100 g of dry sorbent) } \\
\hline$W_{\mathrm{m}}$ & \multicolumn{2}{|c|}{ Monolayer moisture content in g sorbed/100 g of dry sorbent } \\
\hline$C_{\mathrm{g}}$ & \multicolumn{2}{|c|}{$\begin{array}{l}\text { Energy constant related to the difference between the free enthalpy of the pure liquid state water molecules and in the monolayer. } \\
\text { Proportional to the rate between both the escape and the attachment rate constants of the primary sites }\end{array}$} \\
\hline K & \multicolumn{2}{|c|}{$\begin{array}{l}\text { Ratio between the standard vapour pressure of the liquid and the vapour pressure of the sorbate in the secondary (upper) layers. } \\
\text { Proportional to the rate between the escape and the attachment rate constants for all higher layers }\end{array}$} \\
\hline
\end{tabular}

The $\mu$-FTIR was performed with a MIRAS beamline at the ALBA synchrotron ${ }^{35}$ using a Hyperion 3000 Microscope (Bruker) equipped with a $36 \times$ magnification objective and condenser coupled to a Vertex 70 spectrometer (Bruker) with a $50 \mu \mathrm{m} \mathrm{HgCdTe} \mathrm{(MCT)} \mathrm{detec-}$ tor that was continuously purged with $\mathrm{N} 2$ gas. The measuring range was $4000-900 \mathrm{~cm}^{-1}$, and the spectra collection were carried out in a transmission mode of $4 \mathrm{~cm}^{-1}$ resolution, $10 \mu \mathrm{m} \times 10 \mu \mathrm{m}$ aperture dimensions, a step size of $10 \mu \mathrm{m}$ and 128 co-added scans. Due to the inherent high intensity of the samples and impossibility of obtain lower than $5 \mu \mathrm{m}$ homogeneous sections, $10 \times 10$ aperture was chosen. Despite the lower thickness of the average cuticle and some medullas, previous tests showed that the differences between regions were enough to discern between then. The background spectra were collected from a clean area of the CaF2 window every 20 scans.

The OPUS software (Version 7.5, Bruker) was used to obtain an individual spectrum of the cuticle region. Spectra with a huge saturation were discarded as well as spectra with no signal.

Unscrambler X software (Version 10.5, CAMO Software) was used to perform the statistical analysis. Spectra were corrected using the baseline offset, and linear baseline correction provided by the software. Principal component analysis (PCA) was performed using the spectral region of the $\mathrm{CH} 2$ symmetric peak of the cuticle spectra. The score and loadings values, as well as the mean values, were used for data interpretation. ${ }^{36}$ Averaged spectra of all the hair types were analysed using the Unscrambler X software, and the peak position and amplitude of each spectrum $(n=141)$ were determined using Gaussian/Lorentzian fitting via a self-written python code.

\section{6 | Statistical assessment}

Standard deviations were calculated for all mean values. For group comparisons, one-way analyses of variance or the Kruskal-Wallis test were applied. STATGRAPHICS plus 5 (STatpoint Technologies Inc) was used as software. A P-value below .05 was considered to be significant.

\section{3 | RESULTS AND DISCUSSION}

Water uptake and desorption isotherms for the hair were evaluated using the sorption analyser. The shape of the water equilibrium sorption isotherms obtained for the three types of hair is described by a type II isotherm, which shows a small amount of water at a very low $\mathrm{RH}$ and an increase at high RH (Figure $1 \mathrm{~A})$. For native hairs, the maximum water regain value was significantly higher for the Caucasian fibres than the other two fibre types (Figure 1A and Table 2).

By definition, hysteresis is "a lag in the response of a material in reacting to a change in its condition." The moisture sorption hysteresis results for the sorption and desorption process are different. The extent of hysteresis is associated with the nature and state of the sample components. Hysteresis occurs because the keratin protein conformation changes with variations in its degree of hydration. ${ }^{32}$ The keratinized tissues' structure is known to change significantly when moisture absorbs or desorbs from the tissue. ${ }^{37,38}$ Then, these tissues exhibit an important hysteresis degree with very slow thermodynamic changes with hydration.

The degree of hysteresis clearly increases with the humidity until approximately $70 \% \mathrm{HR}$, in which the maximum is reached. All types of hair exhibit similar hysteresis behaviours, although the Asian hair fibres have a higher degree of hysteresis, marked by its maximum values. This result could be due to the slow absorption/desorption recovery of water compared with the other two fibres. The Asian fibre structure seems to be more resistant to hydration changes (Figure 1B).

The GAB model yields values for different parameters: $W_{m}$, the monolayer capacity; $a_{\mathrm{w}}$ the water activity as vapour, relative pressure, $p / p_{0}$, where the secondary water predominates over the monolayer; $W_{c}$, the moisture at a point and $C_{\mathrm{g}}$ and $K$, the energy constants at the monolayer and at the inside layers, respectively, ${ }^{15}$ with the consideration of a good fit of the GAB model at $R^{2}>.997$ (Table 2). The Asian hair fibres present a low water regain at $95 \% \mathrm{HR}$ and the highest value for the energy constant $C_{g}$ at primary sites. This energy parameter could indicate that the Asian hair fibre monolayer is stronger than that of the other hairs, and this water acts as an 
impediment for the absorption more water. In addition, Asian hair fibres had the lowest value for the energy constant $K$ at the deepest layers, and the least number of secondary layers, which could indicate a high desorption. This result could be related to the greater hysteresis at high HR values found in the Asian hair fibres. In contrast, the Caucasian hair fibres have the highest moisture regain, monolayer capacity $\left(W_{m}\right)$ and high water content to the extent that the secondary water began to predominate the monolayer capacity. Consequently, more layers of secondary water with high energy constants for the secondary layers $(K)$ developed. These parameters may indicate that the Caucasian hair has a high content of water retained.

A kinetic evaluation of the moisture uptake and loss is a good method for obtaining detailed information regarding the structural integrity of a particular sample. ${ }^{39}$ Evaluation of the diffusion coefficients of the three ethnic types of hairs confirmed the differences in their keratinized structure (Table 2). An inverse relationship is noted between the diffusion coefficient and the time parameter. More time is required to reach equilibrium for tissues with low water permeability, resulting in a smaller diffusion coefficient. This effect is observed for the different hair fibre types in the present study. Caucasian hairs presented lower diffusion coefficients than Asian, and the African hair fibres had the highest diffusion coefficients. Therefore, it could be concluded that the African fibres possess high permeability compared with the Asian and Caucasian fibres.

It is important to remark that the Caucasian hair fibres that demonstrated the most water in the surface and inner layers of the fibre and the lowest diffusion coefficients and therefore the lowest permeability. Collectively, these parameters may indicate that Caucasian hair fibres have a structure with a high affinity for water but with many chemical and/or stearic impediments, which allows for a large amount of water penetration and absorption that is desorbed more slowly.

It is important to note that the water diffusion in the absorption process is quite similar for the three types of hair fibres that were evaluated. However, diffusion in the desorption process is considerably higher for the African fibres compared with the Asian and Caucasian fibres ( $D_{\mathrm{a}}$ (desorption), Table 2 ). The absorption step is a kinetic process that is highly influenced by the steric impediments of the hair fibre. However, in the desorption step, the differences observed are due to the water-water and water-fibre interactions; therefore, this step provides more reliable results on the structural state of the fibre. The increased diffusion coefficients for African hair fibres indicate increased permeability of this type of hair, with less impediments to desorb water. Permeability is believed to be governed by the cell membrane complex (CMC), which is mainly composed of internal lipids. The lipid bilayers from the other keratinized tissues, such as the stratum corneum from skin, contain fluid zones and non-fluid zones with different permeability. The lipidic component, primarily located in the cuticle region, would largely be responsible for the diffusion and the effects of variation in $\mathrm{RH}$. To that end, cross sections of each sample were evaluated using $\mu$-FTIR analysis to understand the lipid differences between cuticle regions of the ethnic hairs.

In the IR spectra, the $\mathrm{CH}_{2}$ symmetric stretching $\left(\mathrm{CH}_{2}\right.$ sym), with maxima approximately $2850 \mathrm{~cm}^{-1}$, is the main peak that reflects the phase of the lipids and provides information about the lipid amount, chain conformational order and packing. The shift to higher wavenumbers indicates an increase of lipid disordering. A peak position lower than $2850 \mathrm{~cm}^{-1}$ indicates an orthorhombic (OR) lipid chain conformation, values between 2850 and $2852 \mathrm{~cm}^{-1}$ represent a hexagonal (HEX) chain conformation, and a liquid crystalline (LIQ) chain conformation is indicated by wavenumbers higher than $2852 \mathrm{~cm}^{-1}$. 40,41 The cuticle spectra of African, Asian and Caucasian hair fibres were obtained and analysed (55, 54 and 32 different spectra, respectively).

Spectra ( $n=141$ ) were analysed by PCA to check the differences in the $\mathrm{CH} 2$ sym peak among the samples. PCA is a powerful multivariate analytical approach to analyse the correlation between spectroscopic peaks by calculating principal components (PCs). ${ }^{42}$ The results of $\mathrm{PC}$ analysis are represented in the form of a score plot and the corresponding loadings (Figure 2). Each point (score) in the score plot corresponds to one spectrum.

The PCA analysis of the $\mathrm{CH} 2$ sym peak shows differences among the samples related to their origin. Distribution of the points along the PC-1 axis confirms that there is a high variability (94\%) in the lipid amount among the three hair ethnicities (Figure 2B). The loadings plot of PC-1 shows that samples with positive scores for PC-1 have a higher $\mathrm{CH} 2$ sym peak intensity and, consequently, higher lipid amounts $^{43}$ (Figure 2C). The highest amount of lipid is presented in the African hair cuticle, and the Asian hair cuticle has the fewest lipids in that layer. Despite the PC-2 axis identifying only a 5\% difference between the spectra, the Caucasian hair cuticle showed higher values than the other ethnicities (Figure 2B). Spectra with high scores values for PC-2 have peaks shifted to lower wavenumber values, as indicated by the PC-2 loading plot (Figure $2 \mathrm{C}$ ), with peaks with peak crests more oriented at lower frequencies. The PCA shows that Caucasian hair cuticles have layers with higher lipid conformational order, which was also observed in the average spectra after the baseline offset and linear baseline correction of all spectra (Figure 2A).

Guided by the results of the PCA, the $\mathrm{CH} 2$ sym of each spectrum was analysed using Gaussian fitting, to obtain their peak position and the amplitude of the peak (proportional to the amount). The relative low variability in the samples, despite their intrinsic heterogeneity, also suggests the important role of the cuticle lipid amount and order. The results shown in Figure 3 significantly reflect the differences between the amount of lipid in the three cuticles, and the African cuticle presents the highest value ( $0.39 \pm 0.10$ a.u.), followed by the Caucasian cuticle ( $0.19 \pm 0.11$ a.u.) and finally Asian cuticle $(0.09 \pm 0.05$ a.u.). On the other hand, the Caucasian cuticle presents the highest lipidic order $\left(2851.44 \pm 0.85 \mathrm{~cm}^{-1}\right)$, which was significantly lower than the African and Asian peak positions (2852.67 \pm 0.54 and $2852.06 \pm 1.98 \mathrm{~cm}^{-1}$, respectively) in agreement with the PCA results. 
(A)

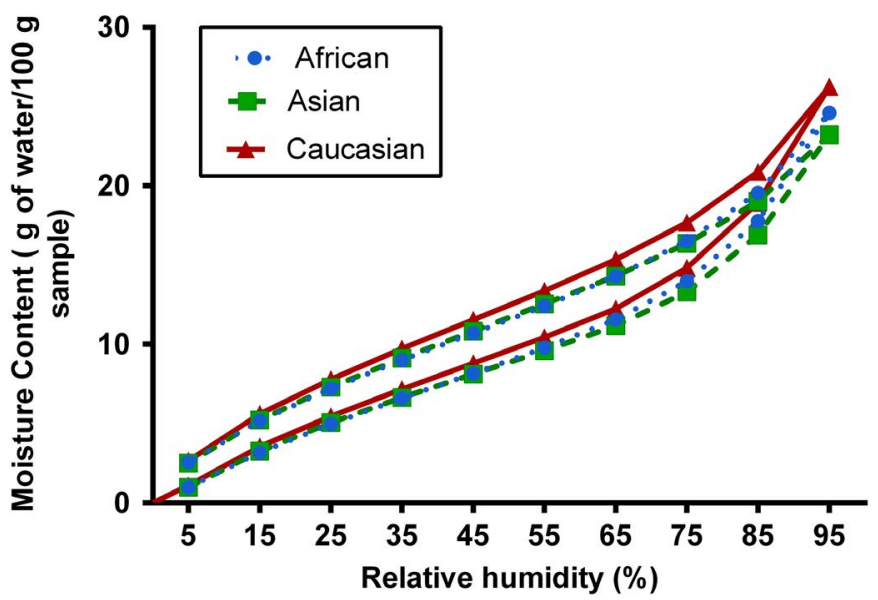

(B) Hysteresis

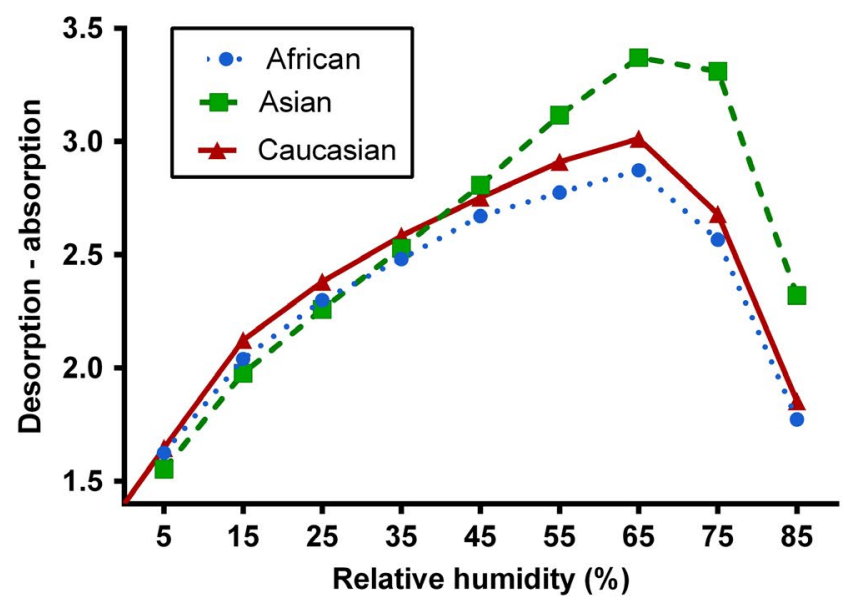

FIGURE 1 Water sorption isotherms and hysteresis curves of native ethnic hairs

The permeability of the fibre was intimately related to the fluidity of the cuticle lipids. Fibres with low position values of the $\mathrm{CH} 2$ sym peaks, such as the Caucasian fibre, have more ordered conformations, thus increasing the barrier function of the cuticle and in agreement with the low diffusion (Table 2). Conversely, African fibres have the lowest lipidic order and the highest diffusion values, despite of the high amount of lipid.

Unlike the ordering, the role and consequences of the lipid amount were more unclear. The amount of cuticle lipid seems to be related to the energy of the primary sites in the acquisition of the water $\left(C_{\mathrm{g}}\right)$. Higher amounts of lipid and low lipidic order, as in the African case, could reduce the interaction between the strength of the water monolayer with the fibre and, therefore, reduce the $C_{g}$.
On the other hand, the low lipid content in the Asian fibre outmost layer could make the primary sites more accessible and increase the energy associated with the monolayer. Furthermore, the lipid components were heterogeneous, including several different families (apolar, free fatty acids, sterols, polar lipids), and their proportion may be more important than the amount. A more accurate study would be necessary to determine the possible effect of the increase in a particular lipid amount on the water dynamics. The study of the cuticle lipids shows that the changes in the resistance of humidity were intimately related with the lipidic order, thereby representing a desirable target to obtain more resistant fibres.

With the purpose to confirm the interactions of the water content with the fibres and examine whether there are some differences

TABLE 2 Parameters of the GAB model: maximum moisture regain GAB monolayer capacity $\left(W_{m}\right)$, water activity as vapour relative pressure $p / p_{0}$, where secondary water predominates over the monolayer $\left(a_{w}\right)$, moisture content at this point $\left(W_{c}\right)$, number of water layers inside the fibres (Layers $\left.n^{\circ}\right)$, GAB energy constant at the monolayer $\left(C_{\mathrm{g}}\right)$, energy constant at the deeper layers $(K)$, GAB determination coefficient $\left(R^{2}\right)$, total time to reach equilibrium $(t)$ apparent diffusion coefficient $\left(D_{a}\right)$ and apparent diffusion coefficient only for desorption process ( $D_{\text {ad }}$ (desorption)) for native ethnic hair fibres

\begin{tabular}{|c|c|c|c|}
\hline & African & Asian & Caucasian \\
\hline Regain at $95 \% \mathrm{HR}$ & $24.60 \pm 0.34^{*}$ & $23.22 \pm 0.66^{*}$ & $26.24 \pm 0.67^{*}$ \\
\hline$W_{m}(g / g)$ & $0.074 \pm 0.001$ & $0.074 \pm 0.007$ & $0.077 \pm 0.004$ \\
\hline$a_{\mathrm{w}}$ & $0.323 \pm 0.002$ & $0.338 \pm 0.014$ & $0.324 \pm 0.003$ \\
\hline$W_{c}(g / g)$ & $0.061 \pm 0.001$ & $0.063 \pm 0.002$ & $0.066 \pm 0.002$ \\
\hline $\mathrm{C}_{\mathrm{g}}$ & $5.264 \pm 0.218$ & $6.051 \pm 1.678$ & $5.915 \pm 0.881$ \\
\hline K & $0.730 \pm 0.010$ & $0.707 \pm 0.014$ & $0.737 \pm 0.017$ \\
\hline$R^{2}$ & $0.999 \pm 0.000$ & $0.997 \pm 0.002$ & $0.998 \pm 0.000$ \\
\hline \multicolumn{4}{|l|}{ Moisture kinetics } \\
\hline
\end{tabular}

${ }^{*}$ Student's $t$ test $P<.05$. 

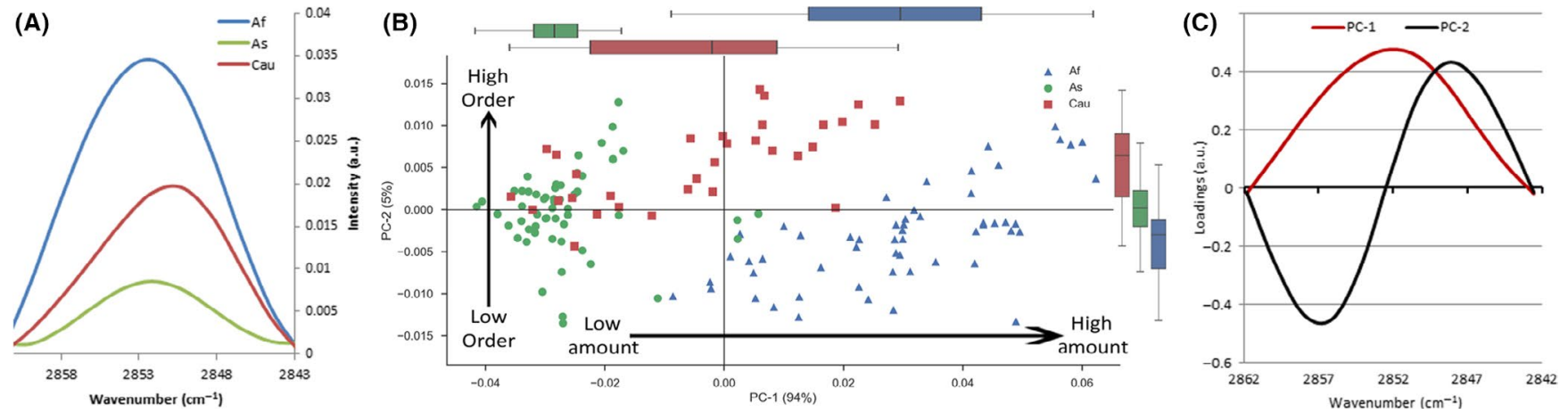

FIGURE 2 Distribution and order analysis of cuticle spectra of hair cross sections using $\mu$-FTIR. A, Mean spectra at $2850 \mathrm{~cm}^{-1}$ for each region. B, PCA score plot of PC-1 vs PC-2 with boxplot for each PC in the opposite site. C, Loading plots of PC-1 and PC-2 of PCA. Boxplot represents $25 / 75 \%$ quintile, whisker $5 / 95 \%$ quantile and central bar represents median

FIGURE 3 Frequencies values and amplitude of the $\mathrm{CH} 2$ symmetric stretching (CH2 Sym). * Dif sig $P<.001$; ${ }^{\dagger}$ Dif sig $P<.0001$
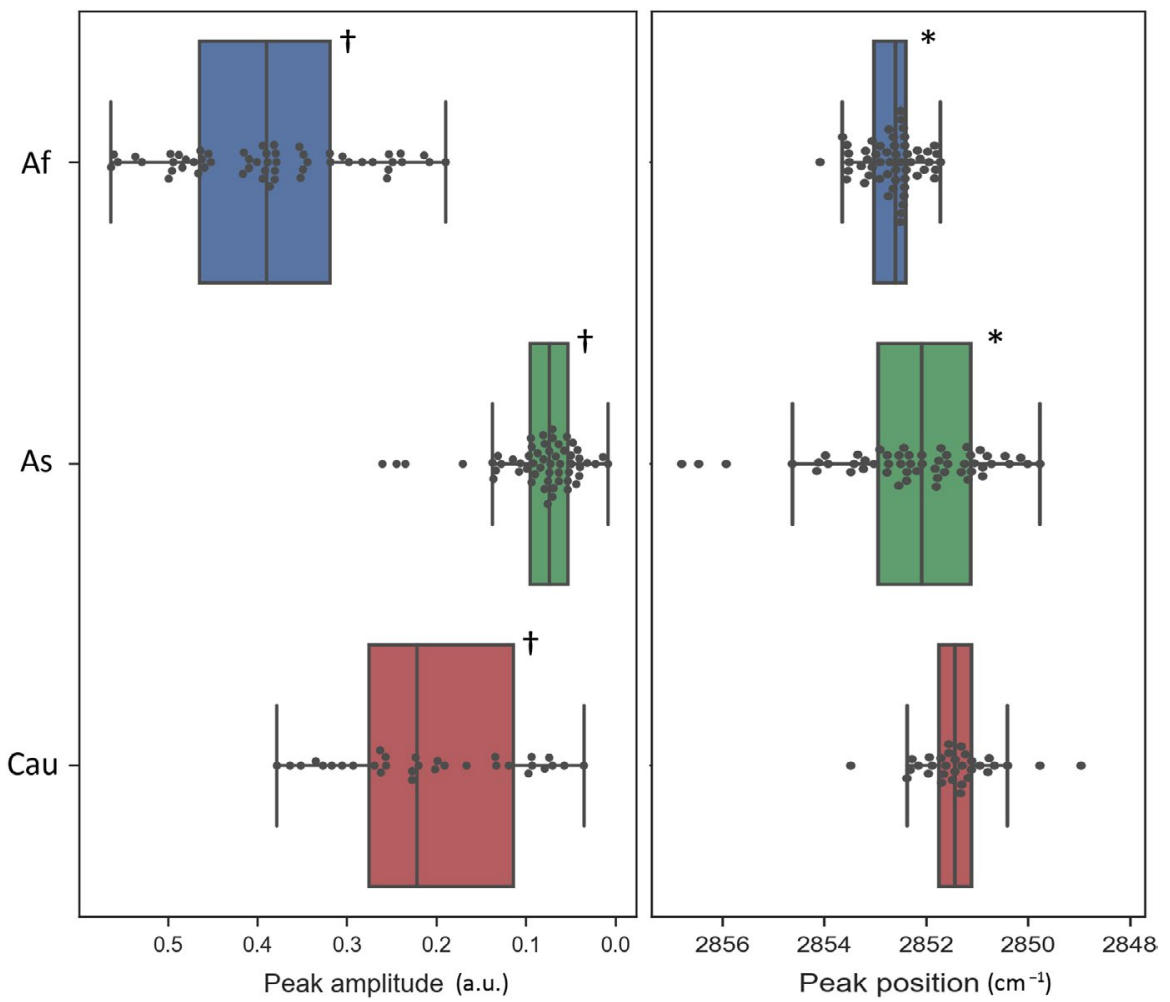

in their thermal stability, a thermoanalytical study was performed. Currently, one of the techniques used to characterize the damage and proposed repair of human hair is differential scanning calorimetry (DSC). Table 3 presents the characteristic peaks of each ethnic hair.

At approximately $120^{\circ} \mathrm{C}$, an endotherm peak is assigned to the evolution of the absorbed water content in the hair. High temperatures indicate that water interactions in the fibres are stronger, and higher enthalpy was correlated with higher amounts. Despite the low differences between the samples, the slight trend between the enthalpy of first peak and the regain value obtained in the DVS analysis (Table 2) was appreciable. The high enthalpy peak of the Caucasian hair $(-191.55 \mathrm{~J} / \mathrm{g})$ and the lower for the Asian hair $(-161.00 \mathrm{~J} / \mathrm{g}) \mathrm{fit}$ with the water content values obtained previously.
There is a second endothermic peak at approximately $233^{\circ} \mathrm{C}$, and the areas (enthalpy) from 5 to $7 \mathrm{Jg}^{-1}$, which strongly correlate with the number of ordered alpha-helices in the fibre. ${ }^{30}$ The third peak from 242 to $244^{\circ} \mathrm{C}$ is due to cystine decomposition. In both peaks, the values are similar among the three different fibres. Some authors found lower denaturation peaks for the African fibres. ${ }^{27}$ Denaturation temperatures normally shift towards higher values with damage (cystine oxidation), whereas the denaturation enthalpy decreases. ${ }^{44}$ Temperatures of both peaks show no difference among the samples, indicating the structural thermostability is similar among the three fibres. Therefore, it can be concluded that no differences in thermal stability related to ethnicity were found in the DSC study, only a slightly higher amount of water in the Caucasian fibre. 
TABLE 3 DSC results of dry ethnic hairs

\begin{tabular}{|c|c|c|c|}
\hline & African & Asian & Caucasian \\
\hline$T\left({ }^{\circ} \mathrm{C}\right)^{*}$ & $117.15 \pm 3.68$ & $120.83 \pm 0.35$ & $120.96 \pm 0.85$ \\
\hline$\Delta H(\mathrm{~J} / \mathrm{g})$ & $-185.24 \pm 63.75$ & $-161.00 \pm 19.32$ & $-191.55 \pm 56.79$ \\
\hline \multicolumn{4}{|c|}{ Amount of ordered alpha-helices } \\
\hline$T\left({ }^{\circ} \mathrm{C}\right)$ & $232.12 \pm 2.02$ & $233.15 \pm 0.59$ & $233.90 \pm 0.95$ \\
\hline \multicolumn{4}{|c|}{ Cystine decomposition } \\
\hline$T\left({ }^{\circ} \mathrm{C}\right)$ & $244.02 \pm 2.37$ & $242.72 \pm 0.35$ & $244.46 \pm 0.85$ \\
\hline$\Delta H(\mathrm{~J} / \mathrm{g})$ & $-8.13 \pm 0.62$ & $-6.635 \pm 2.57$ & $-4.66 \pm 0.45$ \\
\hline
\end{tabular}

Note: Temperature and enthalpy of each peak for the African, Asian and Caucasian hairs.

TABLE 4 TGA results: inflected point temperature $\left({ }^{\circ} \mathrm{C}\right)$ and mass (\%) of each event for the different ethnic hairs

\begin{tabular}{|llll|}
\hline & African & Asian & Caucasian \\
\hline$T\left({ }^{\circ} \mathrm{C}\right)$ & $78.1 \pm 0.5$ & $78.6 \pm 1.3$ & $73.1 \pm 2.0$ \\
\hline Mass (\%) & $7.9 \pm 0.2$ & $8.0 \pm 0.3$ & $9.4 \pm 0.6$ \\
\hline$T\left({ }^{\circ} \mathrm{C}\right)$ & $245.2 \pm 0.0$ & $249.4 \pm 0.4$ & $249.8 \pm 1.6$ \\
\hline Mass (\%) & $8.7 \pm 0.0$ & $8.7 \pm 0.3$ & $8.7 \pm 0.5$ \\
\hline$T\left({ }^{\circ} \mathrm{C}\right)$ & $326.5 \pm 0.2$ & $329.0 \pm 0.0$ & $328.7 \pm 1.5$ \\
\hline Mass (\%) & $50.1 \pm 0.3$ & $48.7 \pm 0.6$ & $49.7 \pm 1.0$ \\
\hline$T\left({ }^{\circ} \mathrm{C}\right)$ & $690.7 \pm 9.7$ & $679.6 \pm 17.9$ & $676.8 \pm 5.6$ \\
\hline Mass (\%) & $31.9 \pm 0.5$ & $33.5 \pm 0.6$ & $31.1 \pm 0.4$ \\
\hline
\end{tabular}

Thermogravimetry is an effective method in the elucidation of probable mechanisms of solid-state reactions such as thermal decomposition and dehydration. Different degradation peaks were obtained for the three fibres. In the hair samples, the first mass loss stage observed can be attributed to water release in the range of $25-170^{\circ} \mathrm{C}$. The second and the third mass loss stages are related to the denaturation of hair keratin, with organic degradation of the hair microfibrils and matrix, at approximately 250 and $330^{\circ} \mathrm{C}$. In the temperature range of $500-900^{\circ} \mathrm{C}$, the complete degradation of the hair keratin carbonic chains occurs ${ }^{44}$ (Table 4).

From the first peak, it is important to remark that the Caucasian fibres present the lowest denaturation temperature $\left(73.1^{\circ} \mathrm{C}\right)$ and the highest amount of water (9.4\%). This is in accordance with the maximum moisture regain and monolayer capacity $\left(W_{m}\right)$ found for this fibre.

The two main peaks in the range of $170-500^{\circ} \mathrm{C}$ could lead to elucidation of the changes in the solid-state reactions such as thermal decomposition of the keratin polypeptide chain denaturation. Even though the mass percentage of these peaks are very similar between ethnicities, it is important to remark that the two peaks of the African ethnic hair present lower temperature thermal decompositions (245, $326^{\circ} \mathrm{C}$ ) than the other fibres $\left( \pm 250, \pm 329^{\circ} \mathrm{C}\right)$. This result means that the African fibres have less cystine disulphide bonds in the matrix, which leads to a decrease in viscosity and a decrease in the protein denaturation. ${ }^{4}$ This effect can be related to the mechanical properties described for these fibres ${ }^{45}$ in which African hair presented the lowest deformation at break, indicating a reduced bonding level of the matrix and lower plasticizer effect in the matrix. Complete thermal decomposition of the fibres occurs at higher temperatures $\left(>650^{\circ} \mathrm{C}\right)$. It is more difficult to make conclusions of this broad peak, as the standard deviation of $T$ is very high. However, it can be remarked that Asian hair fibres experience a higher mass percentage for this degradation peak.

\section{4 | CONCLUSIONS}

Different thermoanalytical and spectroscopic techniques were applied to three ethnic hair fibres with the aim of discerning the structural and chemical differences among them. Water content was determined using different methods: water regain at $95 \% \mathrm{HR}$, diffusion coefficient and GAB monolayer capacity $\left(W_{m}\right)$ by DVS. Lipid amount and order were evaluated using FTIR. Finally, enthalpy of the water evaporation and mass loss were determined using DSC and TGA, respectively.

Dynamic vapour sorption indicates a clearly higher amount of water in the Caucasian hair fibre. This level is reduced in the African fibres and achieves a minimum in the Asian fibres. African fibres show the highest diffusion levels and the Caucasian fibres the lowest ones. The results show that the amount of water content in the fibres is not linked to their diffusion capacity.

The analysis of the lipidic amount and order in the cuticles of different fibres show significant differences among them. The lower order of the African lipidic content could explain their higher diffusion coefficient, despite this fibre having the highest lipid content. The results of the FTIR analysis show the relevance of the lipidic order, but more studies are needed to clarify the effect of the lipidic amount.

It is very difficult to discern protein changes between fibres by thermoanalytical techniques such as DSC or TGA. Slight changes on the water content were observed; however, the results indicate the high similarity in the protein components among the ethnicities. The 
lack of differences observed related to the protein components reinforce the importance of the lipids in the differences observed via the DVS analysis.

These three types of hair clearly differ in a number of important ways regarding permeability. These results may be of interest to chemists and hair care new-product development specialists.

\section{ACKNOWLEDGEMENTS}

The authors wish to thank the 2009 SGR 2012 (AGAUR) for providing support. FTIR microspectroscopy was performed on the MIRAS beamline at the ALBA Synchrotron in collaboration with ALBA staff. Additionally, the authors acknowledge the Service of Dermocosmetic Assessment for its collaboration and the technical support provided by Isabel Yuste.

\section{CONFLICT OF INTEREST}

There are no conflicts to declare.

\section{ORCID}

Marc A. Oliver (iD https://orcid.org/0000-0002-7308-1775

Clara Barba iD https://orcid.org/0000-0002-3084-2925

Meritxell Marti iD https://orcid.org/0000-0001-9681-6466

\section{REFERENCES}

1. Bernard BA. Hair shape of curly hair. J Am Acad Dematol. 2003;48:120-126.

2. Araújo R, Fernandes M, Cavaco-Paulo A. Biology of human hair: know your hair to control it. Adv Biochem Eng Biotechnol. 2010;125:121-143.

3. Coderch L, Mendez S, Barba C, Pons R, Martí M, Parra JL. Lamellar rearrangement of internal lipids from human hair. Chem Phys Lipids. 2008;155:1-6.

4. Jones LN. Hair structure anatomy and comparative anatomy. Clin Dermatol. 2001;19:95-103.

5. Marcott C, Lo M, Kjoller K, et al. Localization of human hair structural lipids using nanoscale infrared spectroscopy and imaging. Appl Spectrosc. 2004;68:564-569.

6. Zhang Y, Senak G, Moore DJ. Measuring changes in chemistry, composition, and molecular structure within hair fibers by infrared and Raman spectroscopic imaging. J Biomed Opt. 2011;6(5):056009.

7. Kamath YK, Hornoby SB, Weigmann HD. Mechanical and fractographic behavior of negroid hair. J Soc Cosmet Chem. 1984;35:21-43.

8. Dekio S, Jidoi J. Hair low-sulphur proteína composition does not differ electrophoretically among different races. J Dermatol. 1990;15:393-396.

9. Dekio S, Jidoi J. Amounts of fibrous and matrix substances in hairs of different races. J Dermatol. 1990;17:62-64.

10. Zhou Y, Rigoletto R, Koelmel D, et al. The effecte of various cosmetic pretreatments on protecting hair form termal damage by hot flat ironing. J Cosmet Sci. 2011;62:265-682.

11. Hassel RL, Hesse ND. Characterization of Water Adsorption and Absorption in Pharmaceuticals. New Castle: TA Instruments; 2007: TA338.

12. Wortmann FJ, Stapels M, Elliot R, Chandra L. The effect of water on the glass transition of human hair. Biopolymers. 2006;81:371-375.

13. Pierlot AP. Water in wool. Text Res J. 1999;69(2):97-103.

14. Rosenbaum S. Solution in water in polymers: the keratin-water isotherm. J Polym Sci. 1970;31C:45-55.
15. Timmermann EO. Multilayer sorption parameters: BET or GAB values? Colloids Surf A Physicochem Eng Asp. 2003;220:235-260.

16. Coderch L, Oliver MA, Martínez V, Manich AM, Rubio L, Martí M. Exogenous and endogenous lipids of human hair. Skin Res Technol. 2017;23(4):479-485.

17. Barba C, Martí M, Carilla J, Manich AM, Coderch L. Moisture sorption/desorption of protein fibres. Thermochim Acta. 2013;552:70-76.

18. Ji JH, Park TS, Lee HJ, et al. The ethnic differences of the damage of hair and integral hair lipid after ultra violet radiation. Ann Dermatol. 2013;25:54-60.

19. Kreplak L, Briki F, Duvault Y, et al. Profiling lipids across Caucasian and Afro-American hair transverse cuts, using synchrotron infrared microspectrometry. Int J Cosmet Sci. 2001;23:369-374.

20. Mansour RSH, Sallam AA, Hamdan II, Khalil EA, Yousef I. Elucidation of penetration enhancement mechanism of Emu oil using FTIR microspectroscopy. Spectrochim Acta A Mol Biomol Spectrosc. 2017;185:1-10.

21. Chan KLA, Kazarian SG, Mavraki A, Williams DR. Fourier transform infrared imaging of human hair with a high spatial resolution without the use of a synchrotron. Appl Spectrosc. 2005;59(2):149-155.

22. Dumas P, Jamin N, Teillaud JL, Millerd LM, Beccard B. Imaging capabilities of synchrotron infrared microspectroscopy. Faraday Discuss. 2004;126:289.

23. Dumas $P$, Millerb L. The use of synchrotron infrared microspectroscopy in biological and biomedical investigations. Vib Spectrosc. 2003;32(1):3-21.

24. Cao J. Melting study of the $\alpha$ form crystallites in human hair keratin by DSC. Thermochim Acta. 1999;335:5-9.

25. Gama RM, Balogh TS, França S, et al. Thermal analysis of hair treated with oxidative hair dye under influence of conditioner agents. J Therm Anal Calorim. 2011;106(2):399-405.

26. Wortmann FJ, Springob C, Sendelbach G. Insvestigations of cosmetically treated human hair by differential scanning calorimetry in water. J Cosmet Sci. 2002;53:219-228.

27. Lima CRRC, Almeida MM, Velasco MVR, Matos JR. Thermoanalytical characterization study of hair from different ethnicities. J Therm Anal Calorim. 2016;123:2321-2328.

28. Éhen ZS, Novák CS, Sztatisz J, Bene O. Thermal characterization of hair using TG-MS combined thermoanalytical technique. J Therm Anal Calorim. 2004;78:427-440.

29. Wendlandt WW. Thermal Analysis, 3rd edn. New York: Wiley; 1986:1.

30. Popescu C, Gummer C. DSC of human hair: a tool for claim support or incorrect data analysis? Int J Cosm Sci. 2016;38:433-439.

31. Tonon RV, Baroni AF, Brabet C, Gibert O, Pallet D, Hubinger MD. Effect of stirring on osmotic dehydration of yellow pitahaya (selenicereus megalanthus S.). J Food Eng. 2009;34(7):492-496.

32. Anderson RB, Hall WK. Modifications of the Brunauer, Emmett and Teller equation II. J Am Chem Soc. 1948;70:1727-1734.

33. Manich AM, Maldonado F, Carilla J, Catalina M. Istoermas de sorción/desorción de humedad en el colágeno. Arnedo: 59 Congreso de la AQEIC; 2010:125-146.

34. Vickerstaff T. The Physical Chemistry of Dyeing. London: Oliver and Boyd; 1954.

35. Yousef L, Ribó A, Crisol I, et al. MIRAS: the infrared synchrotron radiation beamline at ALBA. Synchrotron Radiat News. 2017;30:4.

36. Martin FL, Kelly JG, Llabjani V, et al. Distinguishing cell types or populations based on the computational analysis of their infrared spectra. Nat Protoc. 2010;5:1748-1760.

37. Syed A, Kuhajda A, Ayoub H, Ahmad K, Frank EM. African-American hair: its physical properties and differences relative to Caucasian hair. Cosmet Toil. 1995;110:39-48.

38. Hel Heldman DR, Hall CW, Hedrick I. Vapor equilibrium relationships of dry milk. Dairy Sci. 1965;48:845-852. 
39. Barba C, Martí M, Manich AM, Carilla J, Parra JL, Coderch L. Water absorption/desorption of human hair and nails. Thermochim Acta. 2010;503-504:33-39.

40. Mendelsohn R, Flach CR, Moore DJ. Determination of molecular conformation and permeation in skin via IR spectroscopy, microscopy, and imaging. Biochim Biophys Acta. 2006;923-933.

41. Nishimura K, Nishino M, Inaoka $\mathrm{Y}$, Kitada $\mathrm{Y}$, Fukushima M. Interrelationship between the hair lipids and the hair moisture. J Cosmet Sci Soc Jpn. 1989;13:134-139.

42. Bonnier F, Byrne HJ. Understanding the molecular information contained in principal component analysis of vibrational spectra of biological systems. Analyst. 2012;137:322-332.

43. Schreiver I, Hesse B, Seim C, et al. Synchrotron-based v-XRF mapping and $\mu$-FTIR microscopy enable to look into the fate and effects of tattoo pigments in human skin. Sci Rep. 2017;7:11395.
44. Monteiro VF, Maciel AP, Longo E. Thermal analysis of Caucasian human hair. J Therm Anal Calorim. 2005;79:289-293.

45. Menkart J, Wolfram LI, Mao I. Caucasian hair, Negro hair and wool: similarities and differences. J Cosmet Chem. 1996;7:769-787.

How to cite this article: Oliver MA, Coderch L, Carrer V, Barba C, Marti M. Ethnic hair: Thermoanalytical and spectroscopic differences. Skin Res Technol. 2020;00:1-10. https://doi.org/10.1111/srt.12842 